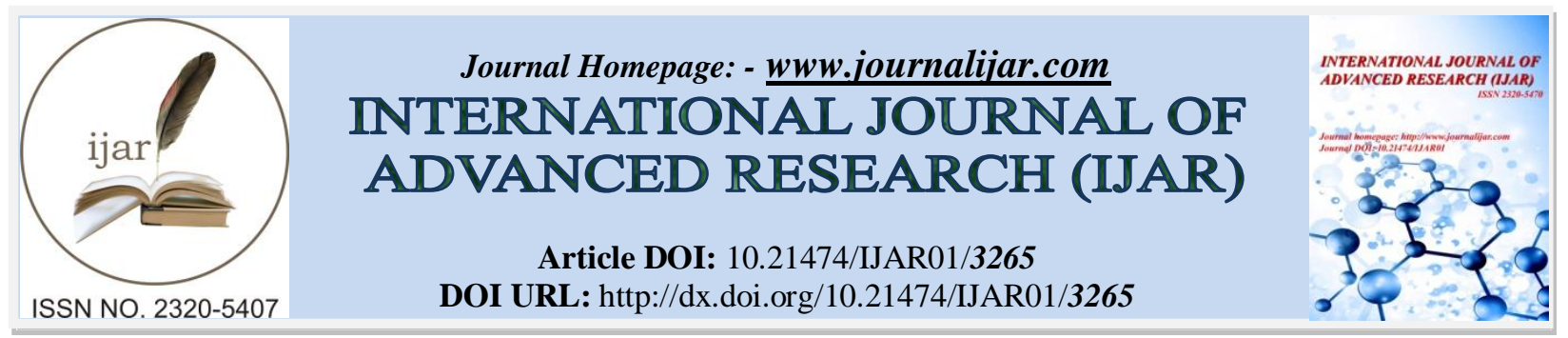

RESEARCH ARTICLE

\title{
PEDIATRIC CASE OF ACCIDENTAL ORAL OVER DOSE OF METHOTREXATE.
}

Mohammed Almadan, Hedayah Hodayah and Ghufran Hodyah.

Pediatrics Department, King Fahad of The University Hospital, Khobar,Kingdomof Saudi Arabia.

\section{Manuscript Info}

Manuscript History

Received: 10 December 2016

Final Accepted: 20 January 2017

Published: February 2017

\section{Abstract}

We present the second youngest reported case of a single oral overdose of methotrexate inotherwise well 19 month sold child. Initialhistory revealed possibleingestion of 10 tablets, eachcontaining $2.5 \mathrm{mg}$. The peakmethotrexate levelwas. $\quad 92 \mathrm{mmol} / \mathrm{L} \quad$ measured8 hourfollowingingestion.

Copy Right, IJAR, 2017,. All rights reserved.

\section{Introduction:-}

paediatriccasesofaccidentaloverdosehasbeen one of the most common causesofinjuryinchildrenless than5yearsof

(1)

age . Althoughpreventive measure hasdecrease the incidence of paediatricoverdose, it continueto occur. Earlyrecognition and treatment is essential toprevent morbidity and mortality. Our caseisan accidental oral overdose ofMTX, the management is not well established,it'smainly basedon experience followingparenteral overdose of methotrexate in children .

\section{Case Report:-}

A19month oldgirlpresentedtothe paediatricemergencyof kingFahadHospital ofthe University2hours aftershewasdiscovered by her mother that she wasplayingwith her grandmother methotrexatetabletsthat hadfallen to the floor, andsome in her mouth. The exactnumber of tabletswas not known, but each tabletcontains $2.5 \mathrm{mgof}$ methotrexate. The total tablet were50, thepossibleingestion of 10 tablets. There was nosymptomsbetween ingestion andpresentationto local hospital. Patient was givenactivated charcoal andsend home , but she presentedto theemergencydepartment. Thechild was otherwisewellwith no significantmedical history. Thechildhad no historyof nausea, vomiting,abdominal discomfort or stoolchanges,andwasnot on any medication .

She appeared wellandalertwith normal observation . Herweight was11.2 kilogram andClinical examinationwasunremarkable . Thepatientwas admitted for observation. A peripheralIntravenouslinewas established and blood sample were sent for routine laboratory studies with determinationof methotrexatelevelsata referencelaboratory. Withserial Laboratoryresult (Table1), the initial methotrexate level was not available from a reference laboratory; however, becausethe patientcouldhavetakenupto $25 \mathrm{mgof}$ methotrexate $(51 \mathrm{mg} / \mathrm{m} 2)$, she was admittedto the paediatricICUformonitoring. Serial laboratory studies and leucovorin therapy givenasneeded. Shewas managed initially with IV hydration with sodium bicarbonate $(40 \mathrm{mg} / \mathrm{l})$ andfolic acidgiven orallyinadoseof $1 \mathrm{mg} /$ day.

Thefollowing morningitwas decided tostart treatmentwith1 0 mgof calcium folinateinfusion (leucovorin )every8 hourswhichisequal to $40 \mathrm{mg}$ /day equivalent to the maximum dose ofmethotrexate ingested ( $1 \mathrm{mg} / \mathrm{mgMTX}$ ). 
Thechildremainedinthepaediatricward whileshe completed72hoursof leucovorin rescue. Laterthat firstday of admission, the methotrexatelevelfrom the initial blood test confirmeda toxic level

$0.92 \mu \mathrm{mol} /$ litre .The doseof calciumfolinatewas10mgsixhourlyon day 2 , and15 mgsixhourlyonday 3 based onthe corresponding methotrexate level.Onday 4 , the methotrexatelevelwaslessthan $0.02 \mu \mathrm{mol} / \mathrm{litre}$ andcalciumfolinate discontinued .

\section{Discussion:-}

Methotrexate(MTX), a folicacid analogueand antagonist,is used inthe treatmentof particular cancers, autoimmune diseases,placenta accrete andectopic pregnancy. MTX binds to the enzyme dihydrofolatereductase (DHFR), inhibiting the formation of reduced folateandthymidylatesynthetase, resultingintheinhibition of de novo thymidylate,purine andprotein biosynthesis.

At oral doses of $<20 \mathrm{mg} / \mathrm{m} 2$, MTXisrapidly absorbed by an active saturabletransport mechanismwithabioavailabilityof 50-95\%, a peak concentration of $\cdot 3-2 \cdot 2 \mathrm{mmol} / \mathrm{lbeing}$ reached within

$1 \cdot 5-2 \cdot 5 \mathrm{~h}$ from intake, and aneliminationhalflifeof $4-6 \mathrm{~h}$, which is dependenton numbers of factors including age , concentration, durationofexposure, andrenal function.

There is greatvariabilityin blood levels, toxicityandresponse amongpatientsreceiving the same dose per weight (5)

or body surface area. This diversity can, be linked to some extent to the sequence of variations in genes

involved in drug absorption, metabolism, excretion, cellular transport, and effector targets or target pathways.

Millimolar concentrations of MTX for minutesorhoursmayleadto acute renal,CNS, and liver toxicity, whereasconcentrations of $0 \cdot 05-0 \cdot 1 \quad \mu \mathrm{mol} / \mathrm{lfor}$ morethan24-48 $\mathrm{h}$ willresultinhaematologicaland (9)

gastrointestinaltoxicity. DNAsynthesisin bone marrow and intestinal epithelium will be inhibited if MTX concentration was greater than $10 \mathrm{mmol} / \mathrm{L}$.

Oral ingestion of MTX results inlittle toxicity. Thismay be attributable to the pharmacodynamics of MTX Toxicityfrom overdose ${ }^{(2)}$ however , as its indicationsfor use increase,more accidentaloverdoses canbe expected. MTX toxicitycan affectmultiple organsystemsincludingbonemarrow, liver, intestinal tract,

kidneys,lungs, skin,andbloodvessels, resultingindeath insevere cases.

The physical side effects of MTX in children are the sameasinadults, though childrengenerallytolerate (8)

MTXwell .'Themost commonside effectsinvolve the gastrointestinaltract, including nausea and vomiting, transient elevation of liver- associated liver enzyme levels, usually occurringwithinthe first 24 hours. Mostcasesare mild, cause nosymptoms, resolve within7 to 10 days, andresult in no permanent liver damage. Largeoverdosecanresultinacute hepatitis". 
Table.1 Result of blood tests including methotrexate levels taken at this times shown post ingestion

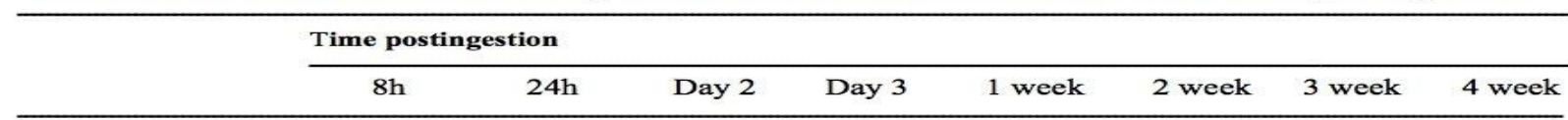

\begin{tabular}{|c|c|c|c|c|c|c|c|c|}
\hline Methotrexate, $\mu \mathrm{mol} / 1$ & 0.92 & 0.04 & 0.01 & 0.01 & Nil & & & \\
\hline Heamoglobin,g/dl (10.5-13.5) & 13.1 & 12.6 & 11.8 & 11.0 & 10.5 & 10.5 & 12.6 & 12.5 \\
\hline White cell count, $\times 10 \times 9 / 1(4.5-15)$ & 13.1 & 11.4 & 9.9 & 10.9 & 10.3 & 9.6 & 10.1 & 8.3 \\
\hline Platelets, $\times 10 \times 9 / 1(150-450)$ & 225 & 237 & 264 & 281 & 320 & 274 & 370 & 294 \\
\hline Nuetrophil, $\times 10 \times 9 / 1(.50-8.00)$ & 28.0 & 55.6 & & 50.1 & 22.0 & 19 & 27 & 25 \\
\hline Lymphocyte, $\times 10 \times 9 / 1(1.50-6.80)$ & 62 & 37.3 & & 43.7 & 64.7 & 74 & 66 & 64 \\
\hline Sodium, mmol/1 (135-145) & 140 & 136 & 137 & 138 & 134 & 138 & 136 & 138 \\
\hline Potassium, mmol/1 (3.6-5.3) & 4.3 & 4.9 & 3.7 & 4.9 & 5.0 & 4.5 & 4.0 & 4.1 \\
\hline (Urea, mmol/1 (1.7-6.7) & 10.0 & 7.0 & 4.0 & 17 & 21 & 14.0 & 9.0 & 7.0 \\
\hline Creatinine, $\mu \mathrm{mol} / 1(8-56)$ & 0.4 & 0.3 & 0.3 & 0.3 & 0.3 & 0.3 & 0.5 & 0.5 \\
\hline Albumin, $\mathrm{g} / 1(35-50)$ & 4.3 & 3.4 & 3.5 & 3.6 & 3.4 & 3.9 & & 3.9 \\
\hline Bilirubin, $\mu \mathrm{mol}(<20)$ & 0.2 & 0.5 & 0.7 & 0.2 & 0.2 & 0.2 & & 0.3 \\
\hline $\begin{array}{l}\text { Alkaline phosphatase, IU/1 } \\
(30-350)\end{array}$ & 375 & 318 & 282 & 255 & 269 & 368 & & 326 \\
\hline Alanine phosphatase, IU/1(5-40) & 57 & 50 & 56 & 34 & 40 & 46 & & 44 \\
\hline GT, IU/1 (7-32) & 16 & 13 & 12 & 15 & 14 & 14 & & 12 \\
\hline LDH & 387 & 326 & 317 & 296 & 384 & 349 & & 282 \\
\hline $\begin{array}{l}\text { Corrected calcium, } \mathrm{mmol} / 1 \\
2.20-2.65))\end{array}$ & 9.5 & 9.6 & & 9.4 & 10.1 & & & 10.0 \\
\hline Phosphate, mmol/1 (1,20-1.70) & 5.7 & 5.1 & & 3.8 & 5.1 & & & 5 \\
\hline
\end{tabular}

Few data existsin theliterature to guide management of oral MTX overdose in childrenortoinformprognosis.

Treatment recommendations for pediatric MTX exposures do not differentiate between routes of exposure. Managementof symptomatic patientsinvolves supportivecare, if available the administration ofantidotes, and the removal of the offendingdrug fromthe body.

Inthis case the patientwas asymptomaticand had ingested unknown amount of MTX. Herinitialserumlevelof 0.92 $\mathrm{mmol} / \mathrm{L}$, reportedafteradmission, was10 times the thresholdfortoxicity.Leucovorin rescueata dosageequal to themaximum amount of MTX ingestedhas beeninitiated alongwith urine alkalisation and diuresis to enhance elimination.Additional care includecontinued leucovorin treatment, activatedcharcoalinERand monitoringof MTX levels.Theavailablerecommendationofmanagingoral overdoseof MTX includethe use ofactivatedcharcoal,gastriclavage, folinicacidrescueand urinaryalkalisation.Thefactors whichareimportantto beaddressed inthemanagementofMTXpoisoningarethetimeofpresentationandseverityoftoxicity

In case series of mainlyadultoral overdoses of methotrexatethere wereno adverseoutcomes in patients where folinicacid rescuewas withheld; however, there isno information regarding ingested amounts or serum methotrexateconcentrationsand istherefore oflimited value.

In the context of MTX poisoning Luecovorinisgivenwithinatimeperiod of 4 hours of the overdose with most of itstherapeutic effectiveness occurringwithinthe first hour of the overdose .Moreover, Theinitialdoseshould beequal or greater than maximumpossible doseofMTXingested. 
The favourable outcomeseen inour patient despite delayedfolinicacid rescuebrings into question the urgencyandthelevelof treatment required followingasingle oraloverdose of methotrexate .Lowto moderate levelofMTXand the limited time of exposure following single oraloverdose of MTX may contribute to the benignoutcomeinthiscase.

There is probablyinsufficient data in children at the current time to avoid intravenousleucovorintherapyandmonitoring for toxicside effects. However ,Supportive care and observation only (6)

should be considered the mainstay of treatment .

\section{Reference:-}

1. Schillie SF, Shehab N, Thomas KE, Budnitz DS. Medication overdoses leading to emergency department visits among children.Am J Prev Med. 2009 ;37(3):181-7.

2. LoVecchioF,Katz,WattsD,etel.Fouryearexperience withmethotrexate exposures . MedToxicol2008;4:149-50.

3. Tuffaha HW, Al Omar S.Glucarpidasefor the treatmentoflife-threatening methotrexateoverdose. Drugs Today(Barc).2012;84:705-11 .

4. OdedraGM,RumackBH(eds):POISONDEX. Englewood,CO:MicromedixInc,1996

5. GibbonBN,MantheyDE

."PediatricCaseofAccidentalOral

OverdoseofMethotrexate".AnneEmergMed1999;34:98- 100 .

6. Hensley MD, Bebarta VS, Borys DJ.A Large Case Series of Acute Pediatric Methotrexate Ingestions.PediatrEmerg Care. 2016 ;32(10):682-684 .

7. MeyerS,EddlestonM,BailyB,DeselH,GottshlingS, GorterL.UnintentionalHouseholdPoisoninginChildren . KlinPediatr.2007;219(5):245-70 .

8. VanderMeerA,WulffraatNM,PrakkenBJ,GijsbersB, RademarkerCM,SinnemaG.PsychologicalSideEffectsofMTXTreatmentinJuvenileIdiopathicArthritis.ClinExpR heumatol.2007;25(3):480-5 .

9. SchmiegelowK.AdvancesinIndividualPredictionof MethotrexateToxicity. BrJHaematol.2009;146(5):489- 503 .

10. Toxbase,UK NatinalPoisonInformationService.hhtp://toxbase.org/Poisons-index-A-Z/MProducts/Methotrexate.

11. ShirazB,SokLK,MuniasamyS."Accidantalmethotrexate ingestionina19-month-oldchild"BMJ Case Rep. 2011;2011. bcr1120103477.

12. Chabner BA, Young RC. Threshold methotrexate concentration for in vivo inhibition of DNA synthesis in normal and tumorous target tissues . J Clin Invest 1973;52:1804-1811 . 